\title{
Effect of 4-h time restricted feeding on body weight, leptin concentration and lipid profile in healthy non- obese male Wistar rats
}

\section{Michael A. Olamoyegun}

Ladoke Akintola University of Technology College of Health Sciences

Folasade 0. Ajao ( $\square$ foajao@lautech.edu.ng )

Ladoke Akintola University of Technology Faculty of Basic Medical Sciences

Marcus O. lyedupe

Ladoke Akintola University of Technology Faculty of Basic Medical Sciences

\section{Research}

Keywords: Time restricted feeding, leptin, body weight, lipid profile

Posted Date: April 7th, 2021

DOl: https://doi.org/10.21203/rs.3.rs-336533/v1

License: (c) This work is licensed under a Creative Commons Attribution 4.0 International License.

Read Full License 


\section{Abstract}

Background: Obesity greatly increases the risk of metabolic diseases and preventive approaches for obesity are often inadequate to effectively prevent and manage the diseases. Altering feeding time strategy intervention decreases caloric intake without calorie counting and may be an effective therapy. Therefore, this study investigates the effect of 4-h time restricted feeding on body weight, leptin concentration and lipid profile in healthy non-obese male Wistar rats.

Methods: Rats placed on time-restricted feeding (TRF) regimen had freely access to food for 4 hour per day at designated periods. Twenty four rats divided into four groups $(n=6)$ were used. Group I animals were placed on a 4 hour per day TRF between 8 am-12noon. Group II rats were also placed on a 4 hour per day TRF between 12noon-4pm. Group III rats also placed on a 4 hour per day TRF between $8 \mathrm{pm}-12$ midnight while Group IV rats had access food and water ad libitum. This diet strategy resembles taking only breakfast, lunch or dinner once a day. The study lasted for a period of 4 weeks with daily food intake and weekly body weight determined throughout the period. At the end of the experimental period, blood glucose, lipid profile and leptin concentration were assessed. SPSS 21.0 package was used for data analysis, one-way analysis of variance (ANOVA) was used to compare the mean values of variables among the groups and bonferroni's posthoc test was used for significance of pair wise comparisons of mean values among the groups. Significance was set at $p<0.05$.

Results: In this study, the body weights and leptin concentrations of 8pm - 12am and ad libitum groups significantly increased compared with the $8 \mathrm{am}-12$ noon and 12 noon $-4 \mathrm{pm}$ groups. Dyslipidemia was observed in the ad libitum group when compared with the 8am - 12noon and 12noon - 4pm groups.

Conclusion: From this study, 4-hr time restricted feeding has beneficial effects on body weight, blood glucose, lipid profile and leptin concentration. This feeding restriction patterns may be helpful in obesity management and in preventing metabolic diseases development in non obese.

\section{Introduction}

Obesity affects one-third of the general population. [1] In 2014, the World Health Organization estimated that there were more than 1.9 billion adult overweight people of whom more than 600 million were obese. [2] More than 115 million people are estimated to have obesity-associated diseases such as cardiovascular disease, stroke, diabetes, dyslipidaemia, obstructive sleep apnea, metabolic syndrome, and breast, colon, and liver cancers. [3]

Changes in diet and exercise are the mains preventive and management approaches for obesity. However, the majority of obese patients are unable to accomplish or sustain intentional weight reduction by diet and exercise alone. [4] Over the last two decades, human and animal studies have shown that timing of meal intake is as important as the composition of the diet and caloric quantity to prevent obesity and its complications [5-8]. Mealtime and cultural eating habits, the quantity, and type of foods ingested can adversely affect health status and increase the likelihood of developing obesity and related 
complications. It has been suggested that time-restricted food intake might be a successful intervention to prevent and manage obesity, metabolic syndrome, and its complications [9-11].

Behavioral weight loss approaches are effective at helping individuals reduce weight by $5-10 \%$ of their initial body weight. $[12,13]$ but a primary public health goal remains prevention of obesity, along with identifying behavioral strategies to optimize weight loss and maintenance. Of the many factors contributing to the obesity epidemic, the timing of food consumption has become the subject of much recent attention for its significant contribution to body weight regulation.[14, 15] Notably, food timing is a potentially modifiable behavioral target, and as such, deserves critical examination for its possible role in achieving weight loss or weight maintenance.

Intermittent fasting (IF) is an increasingly popular dietary approach used for weight loss and overall health. While there is an increasing body of evidence demonstrating beneficial effects of IF on blood lipids and other health outcomes in the overweight and obese, limited data are available about the effect of IF in healthy non- obese animals or humans. This study therefore aimed at investigating the effect of 4 hour time-restricted feeding on body weight, leptin concentration and lipid profile in healthy non-obese male Wistar rats.

\section{Methods}

\section{Animals}

Male Wistar rats (110-140g) were purchased from animal house, Ladoke Akintola University of Technology, Ogbomoso, Oyo State, Nigeria. All animals were housed in ventilated cages on wood chip bedding with a $12 \mathrm{~h}$ light/dark cycle, room temperature of $22^{\circ} \mathrm{C}$ and $40-60 \%$ relative humidity. Rats were acclimatized for a period of 2 weeks before the commencement of the experiment. They also had free access to tap water and were kept on normal diet during the entire study. Body weight was measured every week throughout the experiment. Animal experiments were performed according to the institutional guidelines for the design and statistical analysis of experiments using laboratory animals

\section{Time-restricted feeding}

The rats placed on time-restricted feeding (TRF) regimen had freely access to food for 4hour per day at designated periods as follows - 8am-12noon; 12 noon- $4 \mathrm{pm} ; 8 \mathrm{pm}-12$ midnight for a period of 4 weeks. This diet strategy resembles taking only breakfast, lunch or dinner once a day for a period of 4 weeks.

\section{Experimental design}

Twenty four rats divided into four groups $(n=6)$ were used. Group I animals were placed on a 4 hour per day TRF between 8am-12noon. Group II rats were also placed on a 4 hour per day TRF between 12noon$4 \mathrm{pm}$. Group III rats also placed on a 4 hour per day TRF between $8 \mathrm{pm}-12$ midnight while Group IV rats had access food and water ad libitum. The study lasted for a period of 4 weeks. 
Measurement of body weight and food intake

Body weight of rats was measured weekly using a weighing balance. The percentage (\%) weight gain was calculated as: (body weight on specific week (g) - initial body weight)/initial body weight $\times 100$. Food intake was measured once a week over $24 \mathrm{~h}$ based on the weight of leftover feed out of $160 \mathrm{~g}$ given.

\section{Sample preparation}

At the end of treatment period, the rats were anesthetized with sodium pentobarbital. Blood was collected by cardiac puncture into heparinized bottle and was centrifuged at $3000 \mathrm{~g}$ for 5 minutes. Plasma was stored frozen until needed for biochemical assay.

\section{Biochemical assays}

Rats were fasted for $12 \mathrm{~h}$ before blood glucose measurement. Food was removed from the food dispenser and bedding of the cages was changed to avoid coprophagy. Blood from the tail vein was measured for glucose using the One Touch Basic glucose monitor (Life scan) and reported as $\mathrm{mg} / \mathrm{dl}$. Blood glucose level was measured during the study and prior to sacrifice. Fasting plasma levels of total cholesterol (TC) and triglyceride (TG) were measured by standardized enzymatic colorimetric methods using assay kit obtained from Fortress Diagnostics Ltd. (Antrim, UK). High-density lipoprotein-cholesterol (HDL-C) was measured by enzymatic clearance assay (Daiichi Pure Chemicals Co., Ltd., Tokyo, Japan) whereas low-density lipoprotein-cholesterol (LDL-C) was estimated using modified Friedewald's formula [50]. TC/HDL-C and TG/HDL-C ratios were estimated as marker of atherogenic lipid indices. Plasma leptin was determined using enzyme-linked immunoabsorbent assay (ELISA) kits following manufacturer's instruction (leptin: RayBio® Rat Leptin ELISA Kit, Cat \# ELR-Leptin-001, Norcross, GA, USA).

\section{Data analysis and statistics}

All data were expressed as means \pm SEM. Statistical group analysis was performed with SPSS statistical software (version 21). One-way analysis of variance (ANOVA) was used to compare the mean values of variables among the groups. Bonferroni's posthoc test was used to identify the significance of pair wise comparisons of mean values among the groups. Statistically significant differences were accepted at $p<0.05$.

\section{Results}

Physiological parameters (Body weight, food intake)

The body weight of 8pm - 12am and ad libitum groups significantly increased compared with the 8am 12noon and 12noon -4pm groups (Figure 1\&2).

Blood Glucose 
The fasting blood glucose levels of the normal and the time-restricted fed rats were shown in Figure 3. Fasting glycemia in the 12 noon $-4 \mathrm{pm}$ fed rats increased significantly when compared with the other three groups.

\section{Circulating lipids}

Figure4 depicts the lipid profile of the normal and the time-restricted fed rats. There were significant increases $(p<0.05)$ in the total cholesterol $(T C)$, triglyceride $(T G)$ and very low density lipoprotein cholesterol (VLDL-C)levels of group fed ad libitum when compared with the 8am - 12noon and 12noon $-4 \mathrm{pm}$ groups. There was however no significant difference between the 8pm - 12am and ad libitum TC, TG and VLDL-C levels. For the LDL-C level, there was a significant increase $(p<0.05)$ in the $4 \mathrm{pm}-8 \mathrm{pm}$ rats when compared with the 8 am - 12noon and 12noon $-4 \mathrm{pm}$ rats. There was however a non significant increase in the ad libitum group compared with the 8am - 12noon group. HDL-C levels of the 8pm - 12am and ad libitum groups decreased significantly when compared with the 8am - 12noon and 12noon -4pm groups(Figures4a-e).

\section{Leptin concentration}

Animals in the groups fed ad libitum had significantly elevated leptin concentration $(p<0.001)$ when compared with the other three groups. There was however a non significant increase in the leptin concentration of the 8pm-12am when compared with the 8am - 12noon and 12noon -4pm groups (Figure5).

\section{Discussion}

Intermittent fasting has gained huge recognition over the past decade. Intermittent fasting can be divided into two major subcategories: 1) fasting 1-4 d per week, i.e. alternate day fasting (ADF) or the 5:2 diet [16]; or 2) fasting every day for a 14 to $20 \mathrm{~h}$ period, i.e. time restricted feeding $[17,18]$. This study examined the impact of time restricted feeding (TRF) on body weight and metabolic disease risk factors in healthy non- obese rats. Time-restricted feeding (TRF) is a type of intermittent fasting that involves having a longer fasting period daily. A key point of the TRF approach made use of in the present study is that total daily calorie intake remained the same while the frequency of meals (i.e. time between meals) was altered. This is unlike many other IF regimens, most of which have the goal of reducing total energy intake.

Our data shows that 4 weeks of 4 -h time restricted feeding (i.e. between the hours of 8am-12noon, 12 noon $-4 \mathrm{pm}$ ) resulted in a significant decrease in body weight of the rats in these groups when compared to the group that had access to food ad libitum. This result is however in agreement with other forms of time restricted feeding which reported significant decreases in the body weight of obese and trained athletes exposed to 8-h and 10-h time restricted feeding respectively (A, B, C).Gill and Panda [19] who also examined the effects of 10-h time restricted feeding in overweight healthy adults, showed a $4 \%$ weight loss that was sustained for one year. The significant change observed in body weight of rats 
exposed to 4-h time restricted feeding between the hours of $8 \mathrm{am}-12 \mathrm{noon}, 12 \mathrm{noon}-4 \mathrm{pm}$ for a period of 4 weeks, suggests that non-obese individuals can also not benefit from time restricted feeding in terms of weight loss. Intermittent fasting (IF) of daily fasting for up to 16 hours; or fasting periods of up to 24 hours interspersed with normal feeding days have also been reported to result in weight loss in both humans and laboratory animal models [20].

Dyslipidemia is a condition characterized by abnormal lipid status (such as triglycerides [TG], cholesterol and/or phospholipids) in the blood. It includes elevated blood concentrations of the triad; low density lipoprotein cholesterol (LDL-C), TG, and decreased high-density lipoprotein cholesterol (HDL-C) [21]. Diet is considered to be one of the most important risk factors, which plays a key role in the development of dyslipidemia [22]. Results from this work showed altered circulating plasma lipid concentrations i.e dyslipidemia. There were significant increases in TC, TG, LDL-C and VDLC-C concentrations with a significant decrease in HDL-C concentration in the 8.00pm - 12 midnight and ad libitum rats compared to other regimes of TRF.

Several epidemiological studies have established that dyslipidemia is a major cause of cardiovascular disease $[23,24]$. Altered circulating lipid concentrations are recognized as risk factors for cardiovascular diseases (CVDs) [25]. CVDs are the leading cause of global premature mortality and disability [26]. It has also been shown that an increased level of TC (hypercholesterolemia), particularly LDL-C promotes the atherosclerosis process, leading to the deposition of cholesterol and fatty acids in the artery wall, whilst HDL-C is usually considered to be protective and returns cholesterol to the liver [27, 28]. Epidemiological studies including that of the American Heart Association have also shown that elevated TG levels correlate with elevated CV risk, thus elevated TG levels are an important marker of CV risk [29, 30].

This diet strategy of 4 weeks of 4-h TRF(between the hours of 8am-12noon and 12noon -4pm) suggests cardio-protective effects in non-obese rats, by lowering total cholesterol, triglycerides, LDL and VLDL while increasing HDL cholesterol concentrations. These changes in lipid risk factors are in line with what has been reported for obese alternate day fasting (ADF) subjects [31]. Also, in two ADF studies, triacylglycerols decreased by $15 \%$ after 8 weeks of treatment in obese men and women [32]. Thus, TRF may improve plasma lipids in non-obese subjects as it does in obese subjects. Our results were also confirmed by previous researches suggesting a positive effect of TRF (between the hours of 8am-12noon, 12 noon $-4 \mathrm{pm}$ ) on blood lipid profiles [33 - 35].

Additional vascular benefit including decrease in circulating leptin was also noted in non-obese rats in our diet strategies of TRF compared to the group fed ad libitum. The group fed ad libitum has an outrageous elevated significant leptin level compared to the TRF groups Leptin is a $16 \mathrm{kDa}$ hormone mainly secreted by adipocytes and is involved in the control of food intake via its action on the hypothalamus, leading to the suppression of appetite [36]. Therefore, leptin is an "anorexigenic" hormone. However, obesity is characterized by hyperleptinemia due to the development of leptin resistance [37]. Scarpace and, Zhang (2007) also reported a positive correlation between elevated leptin level and the development of leptin resistance and obesity [38]. Apart from obesity, hyperleptinemia has been also associated with 
hypertension and insulin resistance $[39,40]$. The peripheral actions of leptin include stimulation of inflammatory reaction, oxidative stress, atherogenesis and thrombosis, thus promoting endothelial dysfunction, arterial stiffness, development and vulnerability of atherosclerotic plaques [41-42]. Higher leptin levels have also been considered to be the cause of infertility [43].

Results from this study also suggest that of all the TRF groups, the 8am-12noon, which is a representation of breakfast consumption was the most beneficial since it prevents dyslipidemia, increase in body weight, blood glucose and leptin concentrations. Regular breakfast consumption has been reported to improve several metabolic parameters, mostly associated with cardiovascular risk, reducing circulating LDL cholesterol levels, LDL oxidation and lowers blood triglycerol concentrations [44 - 46]. This also brings to mind the quote of the nutritionist Adelle Davis (in the 1960s), which says: "Eat breakfast like a king, lunch like a prince and dinner like a pauper." (Sifferlin, 2013) [47].

In conclusion, 4-h time restricted feeding has beneficial effects on body weight, blood glucose, lipid profile and leptin concentration and it can be adopt as additional therapy for weight loss management in obese and to protect against development of metabolic diseases in non-obese.

\section{Abbreviations}

TRF: Time-restricted feeding; IF: Intermittent fasting; ADF: Alternate day fasting; TC: Total cholesterol; TG: Triglycerides; HDL-c: High density lipoprotein-cholesterol; LDL-C: Low density lipoprotein-cholesterol; VLDL-c: Very low density lipoprotein-cholesterol; CVDs: Cardiovascular diseases; ELISA: Enzyme-linked immunoabsorbent assay; ANOVA: One-way analysis of variance; SPSS: Statistical package for social Sciences.

\section{Declarations}

\section{Ethics approval:}

All procedure were approved by the Animal care committee of the Ladoke Akintola University of Technology and conducted according to the "Principles of Laboratory Animal Care" and specific national laws where applicable.

\section{Consent to participate:}

Not applicable

\section{Consent for publication:}

Not applicable

\section{Availability of data and Materials:}


The datasets used and/ or analysed during this current study are included in this manuscripts.

\section{Competing interests:}

No competing interests

\section{Funding:}

This research work did not receive any specific funding

\section{Authors' contributions:}

All authors have made considerable contribution to the work and approved the final version of the publication. FO, MA, and MO carried out the experiment. MO wrote the manuscript with the support of FO. FO supervised the project. MA conceived the original idea.

\section{Acknowledgements:}

Not applicable

\section{References}

[1] Flegal KM, Carroll MD, Kit BK, Ogden CL. Prevalence of obesity and trends in the distribution of body mass index among US adults, 1999-2010. JAMA. 2012;307:491-7.

[2] “Obesity and overweight," December 2014, http://www.who. int/mediacentre/factsheets/fs311/en/.

[3] “Controlling the global obesity epidemic," December 2014.

[4] Schuppan D and Schattenberg JM. "Non-alcoholic steatohepatitis: pathogenesis and novel therapeutic approaches," Journal of Gastroenterology and Hepatology, vol. 28, pp. 68-76, 2013.

[5] Aksungar F B, Eren A, Ure S, Teskin O and Ates G. "Effects of intermittent fasting on serum lipid levels, coagulation status and plasma homocysteine levels," Annals of Nutrition \& Metabolism, vol. 49, no. 2, pp. 77-82, 2005.

[6] Norouzy, A., Salehi, M., Philippou E.et al., "Effect of fasting in Ramadan on body composition and nutritional intake: a prospective study," Journal of Human Nutrition and Dietetics, vol. 26, Supplement 1, pp. 97-104, 2013.

[7] Temizhan, A., Tandogan,I., Dönderici, O. and Demirbas, B. "The effects of Ramadan fasting on blood lipid levels,” The American Journal of Medicine, vol. 109, no. 4, pp. 341-342, 2000.

[8] Hatori, M., Vollmers, C., Zarrinpar A.et al., "Time-restricted feeding without reducing caloric intake prevents metabolic diseases in mice fed a high-fat diet," Cell Metabolism, 
vol. 15, no. 6, pp. 848-860, 2012.

[9] A. I. Al-Shafei,“Ramadan fasting ameliorates oxidative stress and improves glycemic control and lipid profile in diabeticpatients,"European Journal of Nutrition, vol. 53, no. 7,pp. 1475-1481, 2014.

[10] A. I. Al-Shafei,"Ramadan fasting ameliorates arterialpulse pressure and lipid profile, and alleviates oxidative stress in hypertensive patients,"Blood Pressure, vol. 23,no. 3, pp. 160-167, 2014.

[11] M. Hatori, C. Vollmers, A. Zarrinpar et al.,"Time-restricted feeding without reducing caloric intake prevents metabolic diseases in mice fed a high-fat diet,"Cell Metabolism,vol. 15, no. 6, pp. 848-860,2012.

[12] Wing RR, Lang W, Wadden TA, Safford M, Knowler WC, BertoniAG, et al. Benefits of modest weight loss in improving cardiovascular risk factors in overweight and obese individuals with type 2 diabetes. Diab Care. 2011;34:1481-6.

[13] National Heart Lung and Blood Institute (NHLBI). Clinical guidelines on the identification, evaluation, and treatment of overweight and obesity in adults: the evidence report: National Institutes of Health. Obes Res. 1998;6Suppl 2:51S-209S. Erratum, Obes Res 1998;6:464.

[14] Bechtold DA, Loudon AS. Hypothalamic clocks and rhythms infeeding behaviour. Trends Neurosci. 2013;36:74-82.

[15] Drapeau V, Gallant AR. Homeostatic and circadian control of food intake: clinical strategies to prevent overconsumption. CurrObesRep. 2013;2:93-103.

[16] Longo VD, Mattson MP. Fasting: Molecular mechanisms and clinical applications. Cell Metab. 2014;19(2):181-92.

[17] Longo VD, Panda S. Fasting, Circadian Rhythms, and Time-Restricted Feeding in Healthy Lifespan. Cell Metab. 2016;23(6):1048-59.

[18] Chaix A, et al. Time-restricted feeding is a preventative and therapeutic intervention against diverse nutritional challenges. Cell Metab. 2014;20(6):991-1005.

[19] Gill S, Panda S. A Smartphone App Reveals Erratic Diurnal Eating Patterns in Humans that Can Be Modulated for Health Benefits. Cell Metab. 2015;22(5):789-98.

[20] Mosley, M.; Spencer, M. A Dieta dos 2 Dias-The Fast Diet; Sextante: Belo Horizonte, Brazil, 2013.

[21] Musunuru K. Atherogenic dyslipidemia: cardiovascular risk and dietary intervention. Lipids. 2010;45:907-14.

[22] Kris-Etherton PM, Binkoski AE, Zhao G, Coval SM, Clemmer KF, Hecker HD, Jacques H, Etherton TD. Dietary fat: assessing the evidence in support of a moderate-fat diet; the benchmark based on lipoprotein 
metabolism. Proc Nutr Soc. 2002;61:287-98.

[23] Panel ED, Grundy SM. An international atherosclerosis society position paper: global recommendations for the management of dyslipidemia. J Clin Lipidol. 2013;7:561-5.

[24] Varady KA, Jones PJ. Combination diet and exercise interventions for the treatment of dyslipidemia: an effective preliminary strategy to lower cholesterol levels? J Nutr. 2005;135:1829-35.

[25] Executive Summary of the Third Report of The National Cholesterol Education Program (NCEP) Expert Panel on Detection, Evaluation And Treatment of High Blood Cholesterol in Adults (Adult Treatment Panel III) JAMA. 2001; 285: 2486-97.

[26] T. Vos, S.S. Lim, C. Abbafati, et al.Global burden of 369 diseases and injuries in 204 countries and territories, 1990-2019: a systematic analysis for the Global Burden of Disease Study 2019. Lancet, 396 (2020), pp. 1204-1222

[27] Klop B, Elte JWF, Cabezas MC. Dyslipidemia in obesity: mechanisms and potential targets. Nutrients. 2013;5(4):1218-40.

[28] Niroumand S, Khajedaluee M, Khadem-Rezaiyan M, Abrishami M, Juya M, Khodaee G, et al. Atherogenic index of plasma (AIP): a marker of cardiovascular disease. Med J Islam Repub Iran. 2015;29:240.

[29] Kasai T, Miyauchi K, Yanagisawa N, et al. Mortality risk of triglyc-eride levels in patients with coronary artery disease.Heart.2013;99(1):22-29.

[30] Sarwar N, Danesh J, Eiriksdottir G, et al. Triglycerides and therisk of coronary heart disease: 10,158 incident cases among262,525 participants in 29 Western prospective studies.Circula-tion. 2007;115(4):450-458.

[31] Varady KA, Bhutani S, Klempel MC, Lamarche B: Improvements in LDL particle size and distribution by short-term alternate day modified fasting in obese adults. Br J Nutr 2011, 105:580-583.

[32] Klempel MC, Kroeger CM, Varady KA: Alternate day fasting increases LDL particle size independently of dietary fat content in obese humans. Eur J ClinNutr 2013.

[33] Bhutani S, Klempel MC, Kroeger CM, Trepanowski JF, Varady KA. Alternate day fasting and endurance exercise combine to reduce body weight and favorably alter plasma lipids in obese humans. Obesity. 2013;21:1370-9.

[34] Klempel MC, Kroeger CM, Bhutani S, Trepanowski JF, Varady KA. Intermittent fasting combined with calorie restriction is effective for weight loss and cardio-protection in obese women. Nutr J. 2012;11:98. 
[35] Klempel MC, Kroeger CM, Varady KA. Alternate day fasting increases LDL particle size independently of dietary fat content in obese humans. Eur J ClinNutr. 2013;67:783 5.

[36] Mechanick JI, Zhao S, Garvey WT. Leptin, An Adipokine With Central Importance in the Global Obesity Problem. Glob Heart. 2017 Dec 13. [Epub ahead of print]

[37] Crujeiras AB, Carreira MC, Cabia B, Andrade S, Amil M, Casanueva FF. Leptin resistance in obesity: an epigenetic landscape. Life Sci 2015; 140: 57-63.

[38] Scarpace PJ, Zhang Y. Elevated leptin: Consequence or cause of obesity? Front Biosci. 2007; 12:3531-44.

[39] Bell BB, Rahmouni K. Leptin as a Mediator of Obesity-Induced Hypertension. Curr Obes Rep 2016; 5: 397-404.

[40] Stepien M, Stepien A, Wlazel RN, Paradowski M, Rizzo M, Banach M, et al. Predictors of insulin resistance in patients with obesity: a pilot study. Angiology 2014; 65: 22-30.

[41] Pérez-Pérez A, Vilariño-García T, Fernández-Riejos P, Martín-González J, Segura-Egea JJ, SánchezMargalet V. Role of leptin as a link between metabolism and the immune system. Cytokine Growth Factor Rev 2017; 35: 71-84.

[42] Beltowski J. Leptin and atherosclerosis. Atherosclerosis 2006; 189: 47-60.

[43] Kamyabi Z, Gholamalizade T: A comparative study of serum and follicular fluid leptin concentrations among explained infertile, unexplained infertile and fertile women. Int J Fertil Steril. 2015, 9:150-156.

[44]. Ruxton $\mathrm{CH}$, Kirk TR. Breakfast: a review of associations with measures of dietary intake, physiology and biochemistry. Br J Nutr 1997; 78: 199-213.

[45] Affenito SG. Breakfast: a missed opportunity. J Am Diet Assoc 2007; 107: 565-9.

[46] Farshchi HR, Taylor MA, Macdonald IA. Deleterious effects of omitting breakfast on insulin sensitivity and fasting lipid profiles in healthy lean women. Am J Clin Nutr 2005; 81: 388-96.

[47] Sifferlin, A., 2013. When to eat breakfast, lunch and dinner. Time, (July 23rd) 〈http://time.com/4408772/besttimesbreakfastlunchdinner/〉.

\section{Figures}




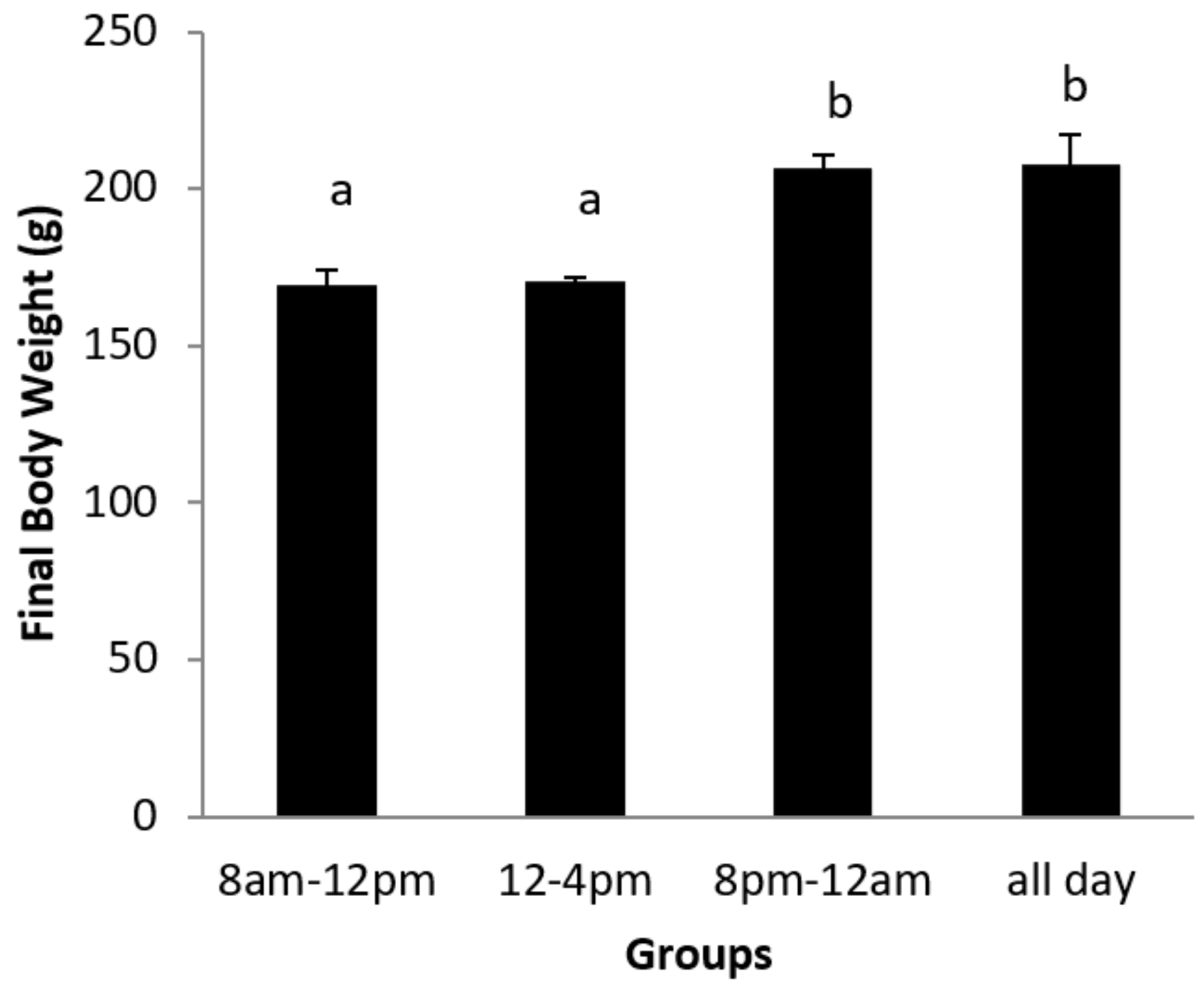

Figure 1

Final body weight 


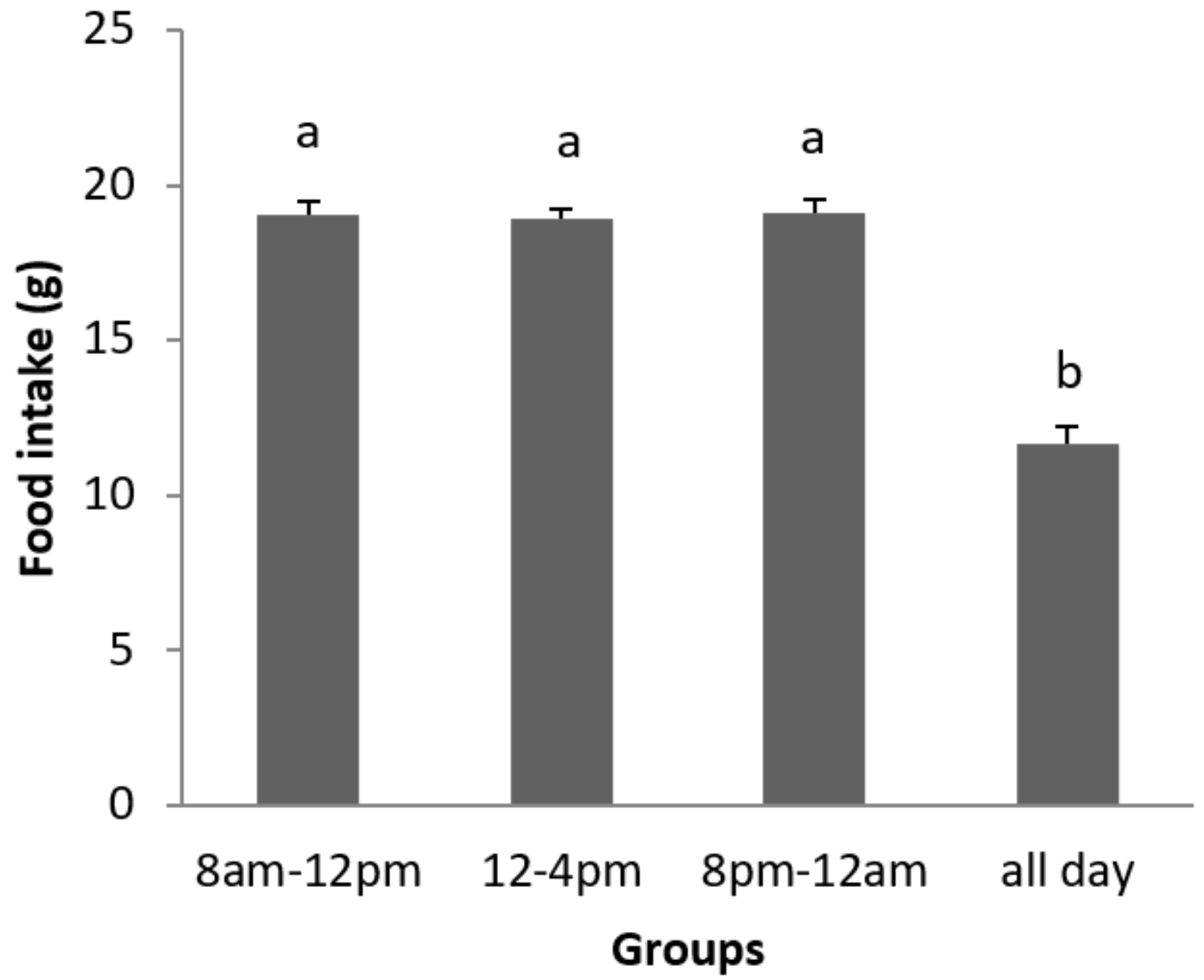

Figure 2

Food intake 


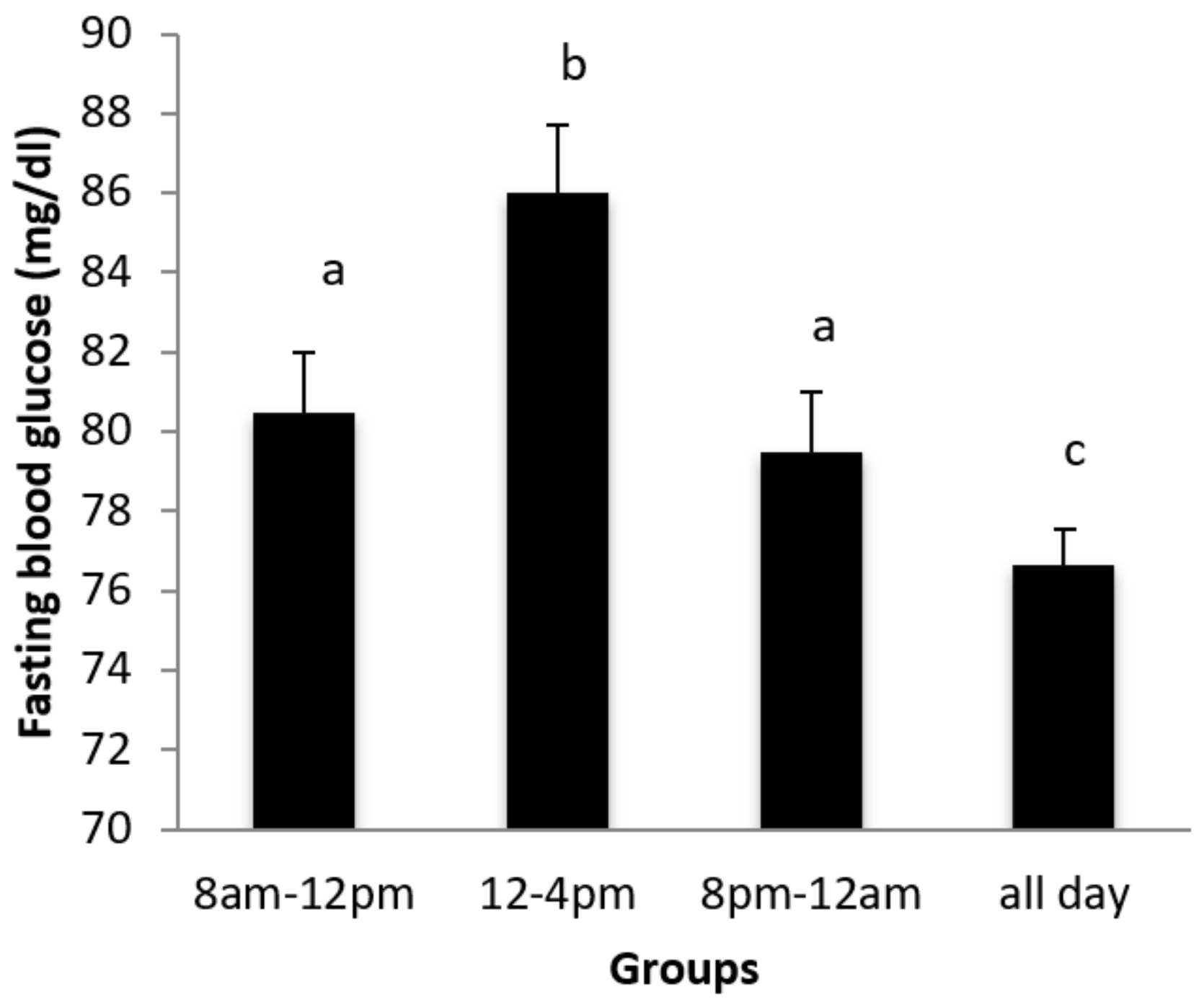

Figure 3

Fasting blood glucose 
$\mathbf{A}$

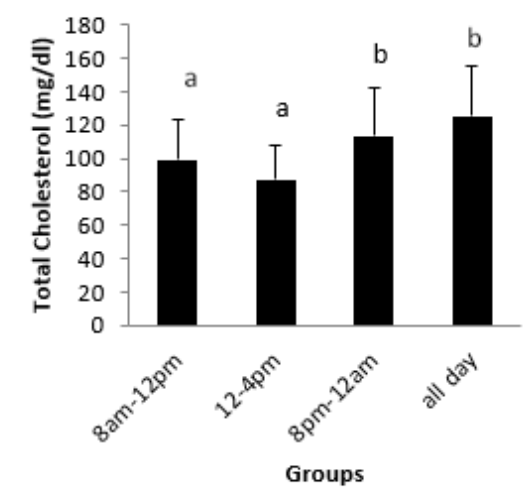

$\mathrm{C}$

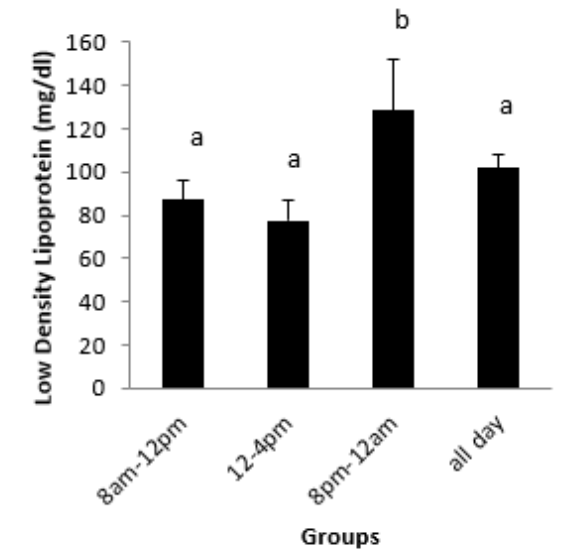

B

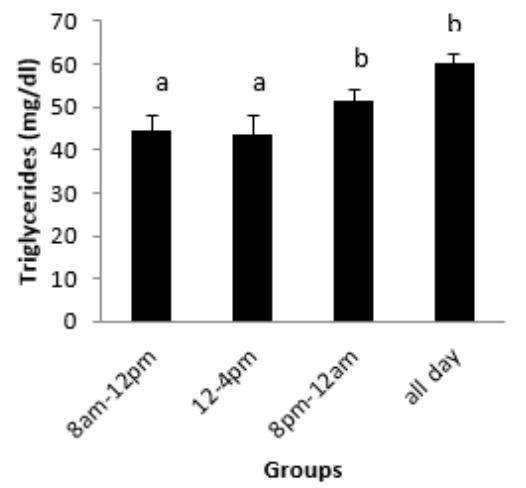

D

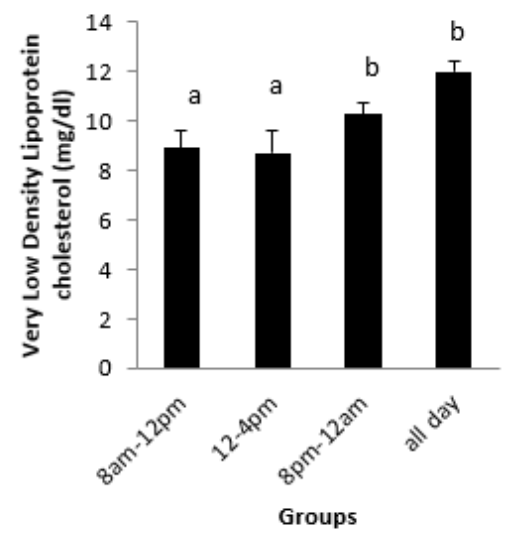

$\mathrm{E}$

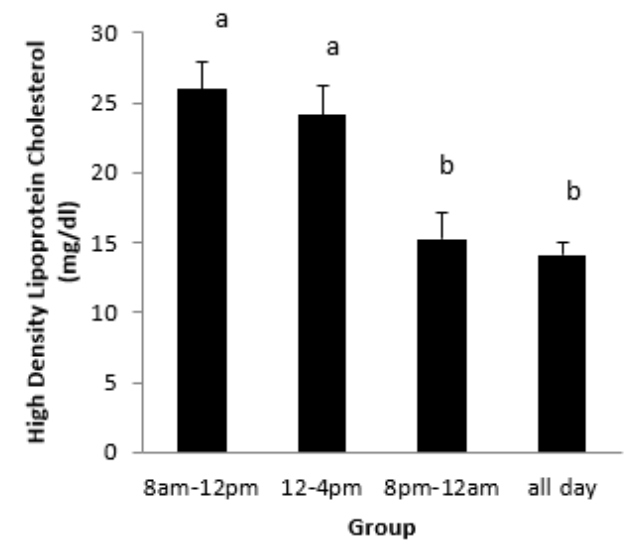

Figure 4

a. Total Cholesterol b. Triglyceride c. Low Density Lipoprotein d. Very Low Density Lipoprotein Cholesterol e. High Density Lipoprotein Cholesterol 


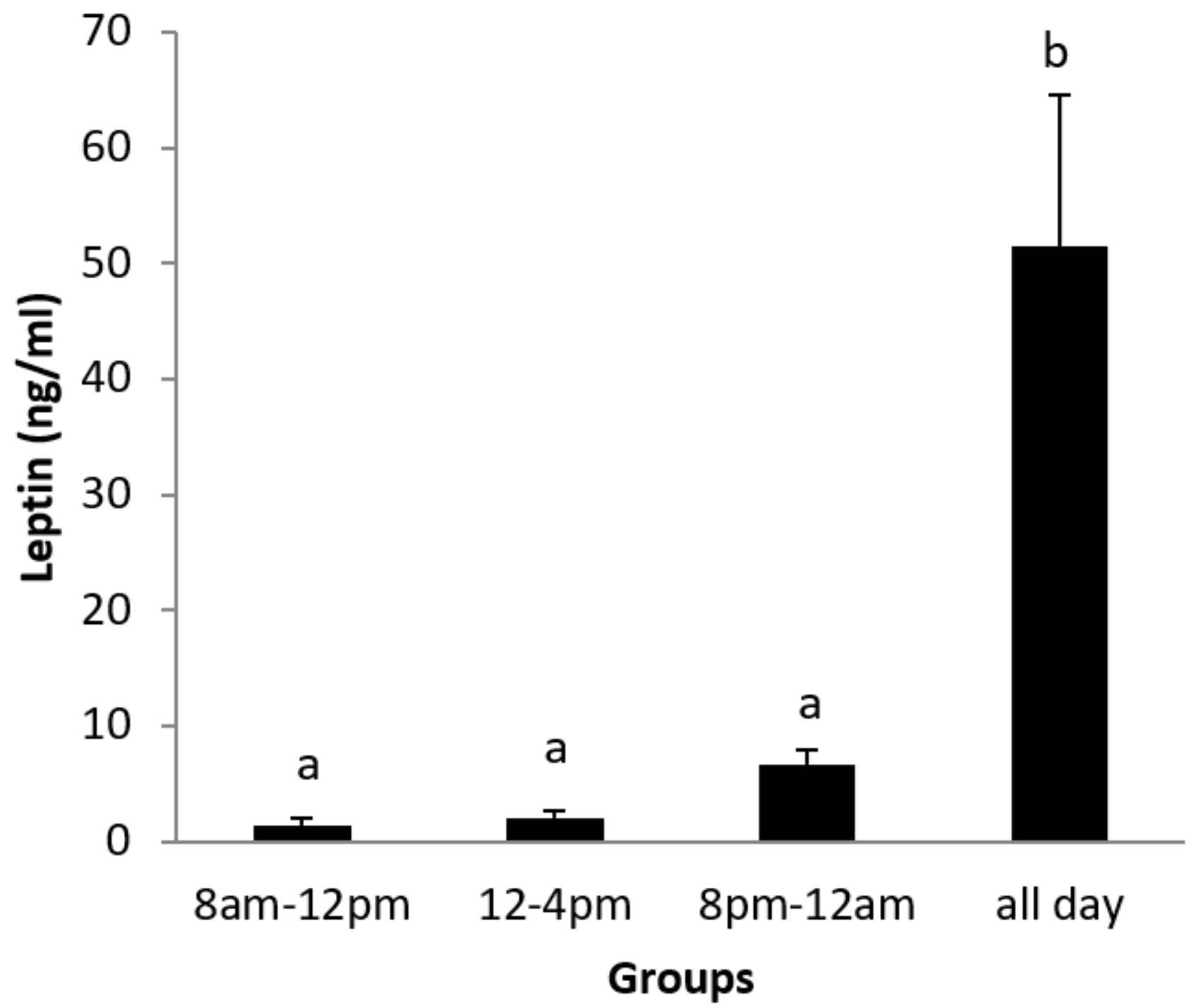

Figure 5

Leptin 